\title{
Factorization for radiative heavy quarkonium decays into scalar Glueball
}

\author{
Ruilin Zhu \\ INPAC, Shanghai Key Laboratory for Particle Physics and Cosmology, \\ Department of Physics and Astronomy, Shanghai Jiao Tong University, \\ Dongchuan RD 800, Shanghai 200240, China \\ State Key Laboratory of Theoretical Physics, \\ Institute of Theoretical Physics, Chinese Academy of Sciences, \\ Zhongguancun E. St. 55, Beijing 100190, China \\ CAS Center for Excellence in Particle Physics, \\ Institute of High Energy Physics, Chinese Academy of Sciences, \\ Yuquan RD 19B, Beijing 100049, China \\ E-mail: rlzhu@sjtu.edu.cn
}

ABSTRACT: We establish the factorization formula for scalar Glueball production through radiative decays of vector states of heavy quarkonia, e.g. $J / \psi, \psi(2 S)$ and $\Upsilon(n S)$, where the Glueball mass is much less than the parent heavy quarkonium mass. The factorization is demonstrated explicitly at one-loop level through the next-to-leading order (NLO) corrections to the hard kernel, the non-relativistic QCD (NRQCD) long-distance matrix elements (LDMEs) of the heavy quarkonium, and the light-cone distribution amplitude (LCDA) of scalar Glueball. The factorization provides a comprehensive theoretical approach to investigate Glueball production in the radiative decays of vector states of heavy quarkonia and determine the physic nature of Glueball. We discuss the scale evolution equation of LCDA for scalar Glueball. In the end, we extract the value of the decay constant of Scalar Glueball from Lattice QCD calculation and analyze the mixing effect among $f_{0}(1370), f_{0}(1500)$ and $f_{0}(1710)$.

KeYwords: QCD Phenomenology, NLO Computations

ArXiv EPrint: 1508.01445 


\section{Contents}

1 Introduction 1

2 Factorization formulae 3

3 Evolution equation for LCDAs of scalar Glueball 6

4 Phenomenological discussions $\quad 8$

5 Conclusion 11

$\begin{array}{ll}\text { A Short-distance coefficients for hard kernels } & 11\end{array}$

\section{Introduction}

Glueball, as a kind of color-confined state with two or more than two gluons, is one of the most important expectation in Quantum Chromodynamics (QCD). The non-Abelian interactions among gluons tell us the existence of Glueball and there is no hidden principle to forbid this kind of state up to now. Many theoretical approaches have been employed to investigate one of the most important quantum numbers of Glueball, i.e. its mass, e.g. Lattice QCD [1-4], QCD Sum Rules [5-11], Supergravity Dual [12], Top-down Holographic Dual [13-15], Rotating Closed Strings [16], MIT Bag Model [17]. In these approaches, the scalar Glueball is expected generally to populate the low enery region from $1 \mathrm{GeV}$ to $2.2 \mathrm{GeV}$, which is also a region rich in $q \bar{q}$ states. A famous criterion to distinguish Glueball from the conventional $q \bar{q}$ states is that the width of Glueball is narrow from the large- $N_{c}$ argument [18-20], where the decay width of the $q \bar{q}$ states is proportional to $1 / N_{c}$ while the width of Glueball is proportional to $1 / N_{c}^{2}$. Another criterion is that Glueball with non-zero spin $(J \neq 0)$ is blind to quark flavor, while scalar Glueball with the quantum number $0^{++}$decays to $q \bar{q}$ is chiral suppressed [21,22] and thus its decay to $s \bar{s}$ is favored than to $u \bar{u}$ or $d \bar{d}$.

The search of Glueball can be carried out in many experiments: $p \bar{p}$ collision, $\pi N$ scattering on polarized/unpolarized targets, central hadronic production, $B$-meson decay, and radiative decays of vector states of heavy quarkonia, i.e. $J / \psi, \psi(2 S)$ and $\Upsilon(n S)$ [23-26]. A great deal of data has been obtained and it is believed that a large possibility of Glueball component in the scalar state $f_{0}(1500)$ or $f_{0}(1710)$ [18]. But a pure Glueball where only gluons contribute the constituent has not been observed or verified up to now. The study of glue-rich processes shall be greatly helpful to hunt the signal of Glueball. The radiative decay of vector states of heavy quarkonia through $V\left(1^{--}\right) \rightarrow G+\gamma$ is one of the most important processes which shall provide a platform to systematically investigate the properties of Glueball. 
In the case of the mass squared of produced Glueball greatly less than that of the parent heavy quarkonium, i.e. $m_{G}^{2} \ll m_{V}^{2}$, a large momentum is transferred, the final Glueball and photon both run in the light-cone, and the light-cone factorization can be well-employed. The soft and collinear physics is only contained in LCDA of Glueball and the NRQCD long-distance matrix elements of the parent heavy quarkonium. The lightcone operators populate the collinear subgroup of four-dimensional conformal symmetry, and the LCDAs of Glueball which incorporate the gluon distribution with certain light-cone momentum fraction can be defined accordingly. The scale dependence of the corresponding physical observable is governed by evolution equation. The evolution equation of LCDAs can be understood as the renormalization group equations for the light-cone operators. Some pioneer works on the evolution equation of distribution amplitudes for exclusive reactions at large momentum transfer can be found in refs. [27-34]. The study of Glueball from radiative Upsilon decay based on soft-collinear effective theory (SCET) can be found in refs. [35, 36].

In the paper, we establish the light-cone factorization for scalar Glueball production in the radiative $J / \psi$ decay, which will be tested at next-to-leading order. The factorization formula is also valid in other vector heavy quarkonium decays, e.g. $\psi(n S)$ and $\Upsilon(n S)$. Through the establishment of the factorization formulae, it is conveniently to perform a systematical phenomenology analysis and open a new and clear widow to investigate the properties of Glueball.

We first give the definition of leading-order LCDAs for scalar Glueball. Since the Glueball and the flavor-singlet $q \bar{q}$ state have identical conformal spin, they will mix each other by renormalization, which is ananogous to the quark-gluon splitting behavior in Proton. Thus we define a two dimensional light-cone distribution amplitude

$$
\boldsymbol{\Phi}(u) \equiv\left(\begin{array}{l}
\phi_{q}(u) \\
\phi_{g}(u)
\end{array}\right),
$$

where the twist-2 LCDAs of $\phi_{i}(u)$ can be written as follows in terms of quark and gluon fields

$$
\begin{aligned}
& \phi_{q}(u)=\int \frac{d z^{-}}{2 \pi} \frac{e^{i(2 u-1) k^{+} z^{-} / 2}}{N_{q}}\left\langle G(k)\left|\bar{\Psi}_{i}\left(-z^{-} / 2\right) L_{i j}\left(-z^{-} / 2, z^{-} / 2\right) \Psi_{j}\left(z^{-} / 2\right)\right| 0\right\rangle, \\
& \phi_{g}(u)=\int \frac{d z^{-}}{2 \pi} \frac{e^{i(2 u-1) k^{+} z^{-} / 2}}{N_{g} u(1-u)} g_{\perp}^{\mu \nu}\left\langle G(k)\left|G^{a,+\mu}\left(-z^{-} / 2\right) L_{a b}\left(-z^{-} / 2, z^{-} / 2\right) G^{b,+\nu}\left(z^{-} / 2\right)\right| 0\right\rangle,
\end{aligned}
$$

where the resummation of all order soft and collinear gluon radiation from quark or gluon field is summarized into the related gauge link, which also ensure the gauge invariant of the defined matrix elements. For a vector $p$, the light-cone component is given by $p^{\mu}=\left(p^{+}, p^{-}, p^{1}, p^{2}\right)$ with $p^{+}=\left(p^{0}+p^{3}\right) / \sqrt{2}$ and $p^{-}=\left(p^{0}-p^{3}\right) / \sqrt{2}$. The $u$ defined above is the momentum fraction in plus direction for one gluon in Glueball. The factor $N_{i}$ satisfies $N_{q}=1$ and $N_{g}=k^{+}$. We define two light-cone vectors $n$ and $\bar{n}$ with $n^{\mu}=(1,0,0,0)$ and $\bar{n}^{\mu}=(0,1,0,0)$ in the light-cone frame. The tensor factor $g_{\perp}^{\mu \nu}$ can be written as $g_{\perp}^{\mu \nu}=g^{\mu \nu}-n^{\mu} \bar{n}^{\nu}-n^{\nu} \bar{n}^{\mu}$. For the gauge link, we have

$$
L(x, y)=P e^{i g \int_{0}^{1} d s(x-y)_{\mu} A^{\mu}((x-y) s+y)},
$$


where $A^{\mu}=A_{a}^{\mu} T^{a}$ in the fundamental representation for the links between quark and anti-quark; while $A^{\mu}=i f^{a b c} A_{b}^{\mu}$ in the adjoint representation for the links between gluon and gluon.

\section{Factorization formulae}

We consider the radiative decay of $J / \psi$ in its rest-frame [37-39]

$$
J / \psi(P) \rightarrow G(k)+\gamma(q),
$$

where the related momenta are given in the brackets. The momentum of Glueball can be written explicitly as $\left(\mathcal{O}\left(k^{+}\right), \mathcal{O}\left(k^{-}\right), \mathcal{O}\left(\Lambda_{\mathrm{QCD}}\right), \mathcal{O}\left(\Lambda_{\mathrm{QCD}}\right)\right)$. Since the charm quark is heavy, the produced Glueball has a momentum of order of $m_{c}$, and satisfies

$$
\frac{k^{-}}{k^{+}}=\frac{m_{G}^{2}}{m_{J / \psi}^{2}} \sim 0.30, \quad \frac{\Lambda_{\mathrm{QCD}}}{k^{+}}=\frac{\sqrt{2} \Lambda_{\mathrm{QCD}}}{m_{J / \psi}} \sim 0.14,
$$

where we assume that $m_{G} \approx 1.7 \mathrm{GeV}, \Lambda_{\mathrm{QCD}} \approx 0.3 \mathrm{GeV}$. If the parent heavy quarkonium becomes to $\Upsilon$, the corresponding ratios of $k^{-} / k^{+}$and $\Lambda_{\mathrm{QCD}} / k^{+}$dramatically decrease to 0.03 and 0.04 respectively. Thus the light-cone factorization is more practical in the $\Upsilon$ radiative decay.

We only consider the QCD corrections here, so the S-matrix element for the decay is

$$
\langle\gamma(q) G(K)|S| J / \psi\rangle=-i e Q_{c} \varepsilon^{* \mu}(q) \int d^{4} x e^{i q \cdot x}\left\langle G(k)\left|\bar{c}(x) \gamma_{\mu} c(x)\right| J / \psi(P)\right\rangle,
$$

where $Q_{c}$ is the electric charge of charm quark, $c(x)$ is the Dirac field for the charm quark, $\varepsilon^{\mu}$ is the polarization vector for the photon. At the leading-order twist defined in eq. (1.3), at least two gluons bound to Glueball. The corresponding contribution to the S-matrix element is

$$
\begin{aligned}
\langle\gamma(q) G(K)|S| J / \psi\rangle= & -i \frac{1}{2} e Q_{c} g_{s}^{2} \varepsilon^{* \alpha}(q) \int d^{4} x d^{4} y d^{4} z e^{i q \cdot x} \\
& \times\left\langle G(k)\left|T\left[\bar{c}(x) \gamma_{\mu} c(x) \bar{c}(y) \gamma \cdot G(y) c(y) \bar{c}(z) \gamma \cdot G(z) c(z)\right]\right| J / \psi(P)\right\rangle,
\end{aligned}
$$

We can calculate the T-ordered operator product by Wick-contraction and we use the expansion of the heavy quark relative velocity $v[40]$

$$
\left\langle 0\left|\bar{c}_{i}(x) c_{j}(y)\right| J / \psi\right\rangle=-\frac{1}{6}\left(P^{+} \gamma_{\mu} P^{-}\right)_{j i}\left\langle 0\left|\chi^{\dagger} \sigma^{\mu} \psi\right| J / \psi\right\rangle e^{-i P \cdot(x+y)}+\mathcal{O}\left(v^{2}\right),
$$

where $\psi$ and $\chi^{\dagger}$ are the NRQCD operators to annihilate the quark and anti-quark respectively. Since the heavy quark relative velocity squared is around 0.3 for $J / \psi$ and 0.1 for $\Upsilon$ [41], we neglect the contribution from higher orders of $v^{2}$ in this paper. By the simplification, the amplitude can be written as

$$
\begin{aligned}
\langle\gamma(q) G(K)|S| J / \psi\rangle= & i \frac{1}{24} e Q_{c} g_{s}^{2} \varepsilon^{* \alpha}(q)(2 \pi)^{4} \delta(P-k-q)\left\langle 0\left|\chi^{\dagger} \sigma^{\beta} \psi\right| J / \psi\right\rangle \\
& \times \int \frac{d^{4} q_{1}}{(2 \pi)^{4}} \Gamma^{\mu \nu}\left(k, q_{1}\right) M_{\alpha \beta \mu \nu}\left(P, k, q_{1}\right),
\end{aligned}
$$


where

$$
\Gamma^{\mu \nu}\left(k, q_{1}\right)=\int d^{4} x e^{-i q_{1} \cdot x-i\left(k-q_{1}\right) \cdot y}\left\langle G(k)\left|G^{a, \mu}(x) G^{a, \nu}(y)\right| 0\right\rangle,
$$

the function $\Gamma^{\mu \nu}\left(k, q_{1}\right)$ incorporates the non-local interactions among two gluons and Glueball, while the function $M_{\alpha \beta \mu \nu}\left(P, k, q_{1}\right)$ is a perturbative kernel, which can be calculated order by order. At twist-2 level the function $\Gamma^{\mu \nu}\left(k, q_{1}\right)$ can be simplified into

$$
\begin{aligned}
\left.\Gamma^{\mu \nu}\left(k, q_{1}\right)\right|_{\text {twist }-2} & =(2 \pi)^{4} \delta\left(q_{1}^{-}\right) \delta^{2}\left(q_{1 \perp}\right) \frac{1}{u(u-1)} g_{\perp}^{\mu \nu} F_{0}(u), \\
F_{0}(u) & =\frac{1}{2 \pi\left(k^{+}\right)^{2}} \int d x^{-} e^{-i(1-2 u) k^{+} x^{-}}\left\langle G(k)\left|G_{+\mu}^{a}\left(-x^{-}\right) G_{+\nu}^{a}\left(x^{-}\right)\right| 0\right\rangle .
\end{aligned}
$$

The naive factorization in eq. (2.6) is valid in tree-level. At NLO and beyond NLO, the factorization should be corrected to including the fluctuation between the gluonium component and the $q \bar{q}$ flavor-singlet component.

Leaving all possible Lorentz invariant construction, the amplitude of $J / \psi$ radiative decays to scalar Glueball can be factorized into

$$
\begin{aligned}
i M= & i e Q_{c} g_{s}^{2}\left\langle 0\left|\chi^{\dagger} \boldsymbol{\sigma} \psi\right| J / \psi\right\rangle \\
& \times \int_{0}^{1} d u \int_{0}^{1} d t\left(\varepsilon_{J / \psi} \cdot \varepsilon_{\gamma} m_{c}^{2} \boldsymbol{H}_{0}(u, v, \mu)+\varepsilon_{J / \psi} \cdot q \varepsilon_{\gamma} \cdot P \boldsymbol{H}_{1}(u, v, \mu)\right) \boldsymbol{\Phi}(t, \mu),
\end{aligned}
$$

with

$$
\begin{aligned}
\left\langle 0\left|\chi^{\dagger} \boldsymbol{\sigma} \psi\right| J / \psi\right\rangle & =\Gamma_{J / \psi}(v, \mu)\left\langle 0\left|\chi^{\dagger} \boldsymbol{\sigma} \psi\right| J / \psi\right\rangle^{r} \\
\boldsymbol{\Phi}(t, \mu) & =\boldsymbol{\Gamma}(u, t, \mu) \boldsymbol{\Phi}^{r}(t, \mu)
\end{aligned}
$$

Note that $\left\langle 0\left|\chi^{\dagger} \boldsymbol{\sigma} \psi\right| J / \psi\right\rangle^{r}$ here is the matrix element after renormalization, which is isolated to the renormalization of LCDAs for Glueball. $\Gamma_{J / \psi}$ is the renormalization factor of LDME for $J / \psi . \Gamma$ is the renormalization factor with $2 \times 2$ matrix elements, which can be calculated through the renormalization of LCDAs of Glueball. $\varepsilon_{J / \psi}$ and $\varepsilon_{\gamma}$ are the polarization vectors of $J / \psi$ and the radiated photon, respectively. $\boldsymbol{H}_{i}$ is the hard kernel with two components. The factorization formula can also employed to $\Upsilon \rightarrow \gamma+G$ by replacing $Q_{c} \rightarrow Q_{b}, m_{c} \rightarrow m_{b}$, $\varepsilon_{J / \psi} \rightarrow \varepsilon_{\Upsilon}$, and $m_{J / \psi} \rightarrow m_{\Upsilon}$.

The typical Feynman diagrams at both tree and one-loop level contributing to the hard kernel for a heavy quarkonium radiative decays to Glueball can be found in figure 1 . Other 73 diagrams for $g g \gamma$ final states can be obtained by exchanging the outgoing gluons or inverting the quark line in figure 1. For tree level, other 3 symmetrical diagrams can be obtained by inverting the direction of the quark in the first line. For one-loop level, there are another 5, 3, and 1 pentagon diagrams respectively compared with the typical diagrams in the second line. There are another 11, 7, 3, and 0 box diagrams respectively in the third line. And there are another 17, 11, and 1 triangle diagrams, another 11 self-energy diagrams in the fourth line. While the diagrams in the fifth line denotes the contribution to $q \bar{q} \gamma$ final states, and another 3 box diagrams and 1 pentagon diagrams are not shown. 


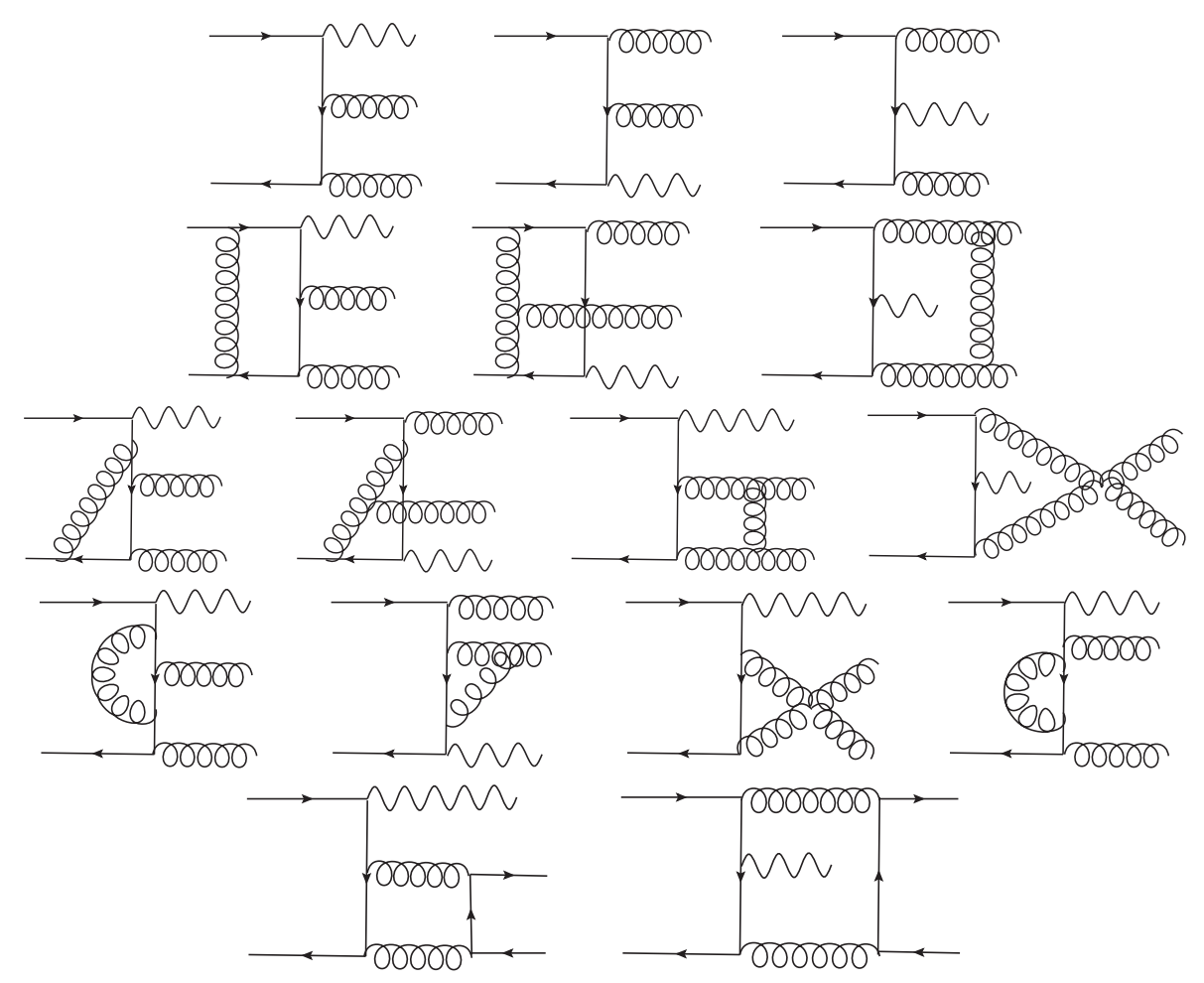

Figure 1. Typical Feynman diagrams for the hard kernel of a heavy quarkonium radiative decays to Glueball.

After the calculation, we can easily get the LO hard kernel

$$
\boldsymbol{H}_{0}^{(0)}=\left(0,-\frac{\sqrt{2}}{N_{c} m_{c}^{3} \sqrt{m_{J / \psi}} u(1-u)}\right), \quad \boldsymbol{H}_{1}^{(0)}=\left(0, \frac{1}{N_{c} m_{c}^{3} \sqrt{2 m_{J / \psi}} u \bar{u}}\right) .
$$

The NLO hard kernel can also be obtained after considering the field renormalization and counter-term. It can be written as

$$
\begin{aligned}
\boldsymbol{H}_{0}^{(1)}= & \frac{1}{\epsilon} \frac{\sqrt{2} \alpha_{s}}{2 \pi N_{c} m_{c}^{3} \sqrt{m_{J / \psi}} u \bar{u}}\left(\frac{n_{f}(2 u-1)(u \ln u+\bar{u} \ln \bar{u})}{2 u \bar{u}}-\epsilon n_{f} H_{0}^{a},\right. \\
& \left.\frac{C_{A}\left(2 u^{2}-2 u+1\right)(u \ln u+\bar{u} \ln \bar{u})}{u \bar{u}}+\frac{\beta_{0}}{2}+\frac{C_{F}}{4 v}\left(\pi^{2} \epsilon-i \pi\left(\frac{\mu}{2 m_{c} v}\right)^{2 \epsilon}\right)-\epsilon H_{0}^{b}\right), \\
\boldsymbol{H}_{1}^{(1)}= & -\left.\frac{1}{2} \boldsymbol{H}_{0}^{(1)}\right|_{H_{0}^{a} \rightarrow H_{1}^{a}, H_{0}^{b} \rightarrow H_{1}^{b}},
\end{aligned}
$$

where the coefficients $H_{0}^{a}, H_{0}^{b}$ which contribute to the finite term is presented in the appendix.

The renormalization factor $\Gamma_{J / \psi}$ can be obtained by the renormalization of the naive LDMEs $\left\langle 0\left|\chi^{\dagger} \boldsymbol{\sigma} \psi\right| J / \psi\right\rangle$. At one-loop level, it can be written as [41]

$$
\Gamma_{J / \psi}^{(0)}=1, \quad \Gamma_{J / \psi}^{(1)}=\frac{\alpha_{s} C_{F}}{4 \pi v}\left(\pi^{2}-i \pi\left(\frac{1}{\epsilon}+\ln \frac{\mu^{2}}{4 m_{c}^{2} v^{2}}\right)\right) .
$$


The factor $\boldsymbol{\Gamma}$ can also be obtained by the renormalization of LCDAs for Glueball. Calculating the LCDAs defined in eq. (1.1), we can easily get the tree-level result

$$
\boldsymbol{\Gamma}^{(0)}(u, t, \mu)=\left(\begin{array}{cc}
\delta(u-t) & 0 \\
0 & \delta(u-t)
\end{array}\right) .
$$

At one-loop level, the related Feynman diagrams can be found in figure 2, and we have

$$
\Gamma^{(1)}(u, t, \mu)=\frac{\alpha_{s}}{2 \pi}\left(\frac{\mu}{\mu_{0}}\right)^{2 \epsilon} \frac{1}{\epsilon}\left(\begin{array}{cc}
S_{q q}^{(1)} & S_{q g}^{(1)} \\
S_{g q}^{(1)} & S_{g g}^{(1)}
\end{array}\right),
$$

with

$$
\begin{aligned}
S_{q q}^{(1)}(u, t)= & C_{F} \frac{u}{t}\left(1+\frac{1}{t-u}\right)_{+} \theta(t-u)+(u \rightarrow \bar{u}, t \rightarrow \bar{t}), \\
S_{q g}^{(1)}(u, t)= & 2 n_{f} T_{F} \frac{u}{t^{2} \bar{t}}(2 u-t-1) \theta(t-u)-(u \rightarrow \bar{u}, t \rightarrow \bar{t}), \\
S_{g q}^{(1)}(u, t)= & C_{F} \frac{u}{t}(2 t-u) \theta(t-u)-(u \rightarrow \bar{u}, t \rightarrow \bar{t}), \\
S_{g g}^{(1)}(u, t)= & C_{A} \frac{u^{2}}{t^{2}}\left(\frac{1}{(t-u)_{+}}+2(\bar{u}+t(1+2 \bar{u}))\right) \theta(t-u) \\
& +\frac{\beta_{0}}{2} \delta(u-t)+(u \rightarrow \bar{u}, t \rightarrow \bar{t}),
\end{aligned}
$$

where $\beta_{0}=11 N_{c} / 3-2 n_{f} / 3$, the group factors $N_{c}=C_{A}=3, C_{F}=4 / 3$ and $T_{F}=1 / 2$ for $S U_{c}(3)$, and the plus function is defined as

$$
F(x, y)_{+}=F(x, y)-\delta(x-y) \int_{0}^{1} d z F(z, y) .
$$

Note that the evolution kernel of LCDA is silimlar to that of the corresponding nonlocal operators, the latter ones have been investigated in refs. [32, 42, 43].

\section{Evolution equation for LCDAs of scalar Glueball}

The study of the universality of LCDAs for Glueball which describes the long-distance interactions effects is a crucial and also interesting issue [44-46]. One can find that all the divergences are cancelled out between the hard kernel and the renormalization factors for both LCDAs and NRQCD LDMEs. We now turn to the scale evolution of LCDAs for Glueball. The reason is that logarithms of the form $\left(\alpha_{s} \ln \left(m_{c, b}^{2} / \mu_{0}^{2}\right)\right)^{n}$, where $\mu_{0} \sim 1 \mathrm{GeV}$ denotes the scale at which nonperturbative physics of the LCDAs exists, are large and must be resummed to all orders. By the renormalization equation, we can resum these large logarithms. The scale dependent equation of LCDA for Glueball reads

$$
\mu^{2} \frac{\partial}{\partial \mu^{2}} \mathbf{\Phi}\left(u, \mu^{2}\right)=\boldsymbol{V}\left(u, t, \alpha_{s}\left(\mu^{2}\right)\right) \otimes \mathbf{\Phi}\left(t, \mu^{2}\right),
$$

where the evolution kernel $\boldsymbol{V}$ is

$$
\boldsymbol{V}=-\boldsymbol{\Gamma}^{-1} \otimes\left(\mu^{2} \frac{\partial}{\partial \mu^{2}} \boldsymbol{\Gamma}\right)=\frac{\alpha_{s}\left(\mu^{2}\right)}{2 \pi}\left(\begin{array}{cc}
S_{q q}^{(1)} & S_{q g}^{(1)} \\
S_{g q}^{(1)} & S_{g g}^{(1)}
\end{array}\right)+\mathcal{O}\left(\alpha_{s}^{2}\right) .
$$



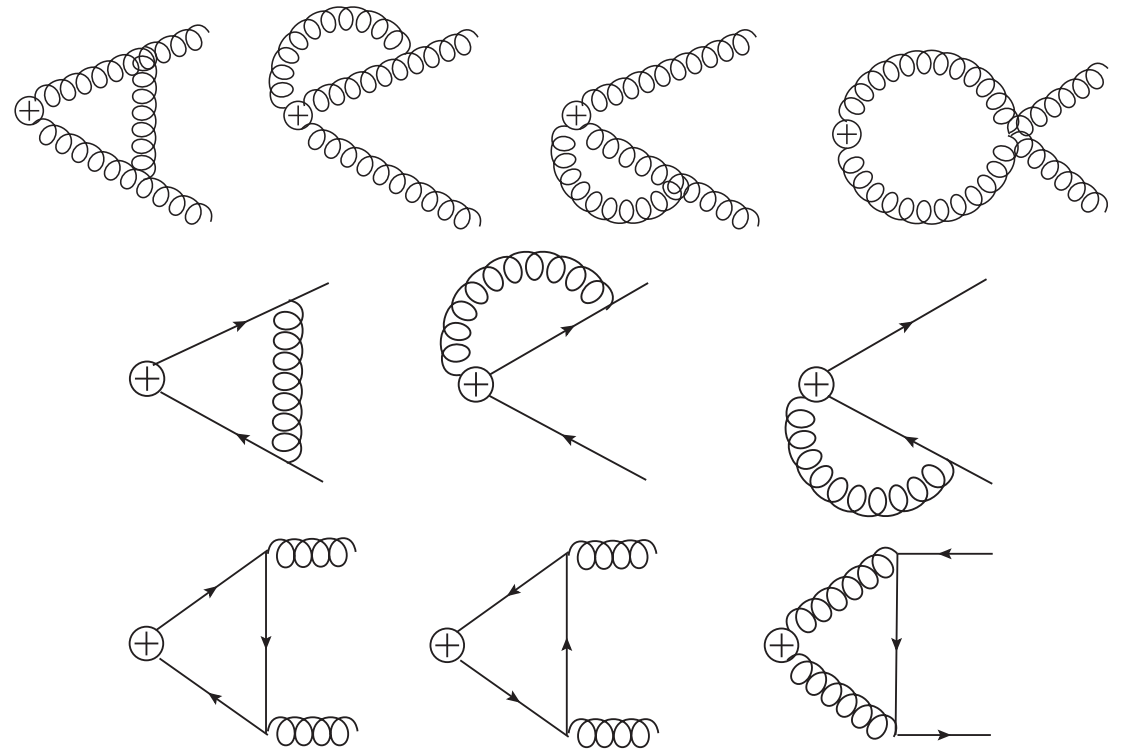

Figure 2. The renormalization of LCDAs for Glueball at one-loop level.

The eigenfunctions of eq. (3.1) after the renormalization are the Gegenbauer polynomials and hence the LCDAs of Glueball $\mathbf{\Phi}\left(u, \mu^{2}\right)$ possess the expansion [35, 36, 47]

$$
\begin{aligned}
& \Phi_{q}\left(u, \mu^{2}\right)=6 u(1-u) f_{q} \sum_{n=1,3, \ldots} a_{n}^{q}\left(\mu^{2}\right) C_{n}^{3 / 2}(2 u-1), \\
& \Phi_{g}\left(u, \mu^{2}\right)=30 u^{2}(1-u)^{2} f_{g}\left(1+\sum_{n=3,5, \ldots} a_{n}^{g}\left(\mu^{2}\right) C_{n-1}^{5 / 2}(2 u-1)\right),
\end{aligned}
$$

where we omit the even $n$ series as a consequence of the symmetry of LCDA for scalar Glueball, i.e. $\Phi_{g}\left(u, \mu^{2}\right)=\Phi_{g}\left(1-u, \mu^{2}\right)$ and $\Phi_{q}\left(u, \mu^{2}\right)=-\Phi_{q}\left(1-u, \mu^{2}\right)$. The Gegenbauer momentum $a_{n}$ also obey the renormalization group equation

$$
\mu^{2} \frac{\partial}{\partial \mu^{2}}\left(\begin{array}{c}
a_{n}^{q}\left(\mu^{2}\right) \\
a_{n}^{g}\left(\mu^{2}\right)
\end{array}\right)=\frac{\alpha_{s}\left(\mu^{2}\right)}{2 \pi}\left(\begin{array}{cc}
\gamma_{n}^{q q} & \gamma_{n}^{q g} \\
\gamma_{n}^{g q} & \gamma_{n}^{g g}
\end{array}\right)\left(\begin{array}{c}
a_{n}^{q}\left(\mu^{2}\right) \\
a_{n}^{g}\left(\mu^{2}\right)
\end{array}\right),
$$

where the anomalous dimensions that govern the evolution of the LCDAs are

$$
\begin{aligned}
\gamma_{n}^{q q} & =C_{F}\left(3+\frac{2}{(n+1)(n+2)}-4 \psi(n+2)-4 \psi(1)\right), \\
\gamma_{n}^{q g} & =\frac{24 n_{f} T_{F}\left(n^{2}+3 n+4\right)}{n(n+1)(n+2)(n+3)}, \\
\gamma_{n}^{g q} & =\frac{C_{F}\left(n^{2}+3 n+4\right)}{3(n+1)(n+2)} \\
\gamma_{n}^{g g} & =C_{A}\left(-4 \psi(n+2)+4 \psi(1)+\frac{\beta_{0}}{2 C_{A}}-\frac{8\left(n^{2}+3 n+3\right)}{n(n+1)(n+2)(n+3)}\right),
\end{aligned}
$$

where $\psi(x)$ is the digamma function. 


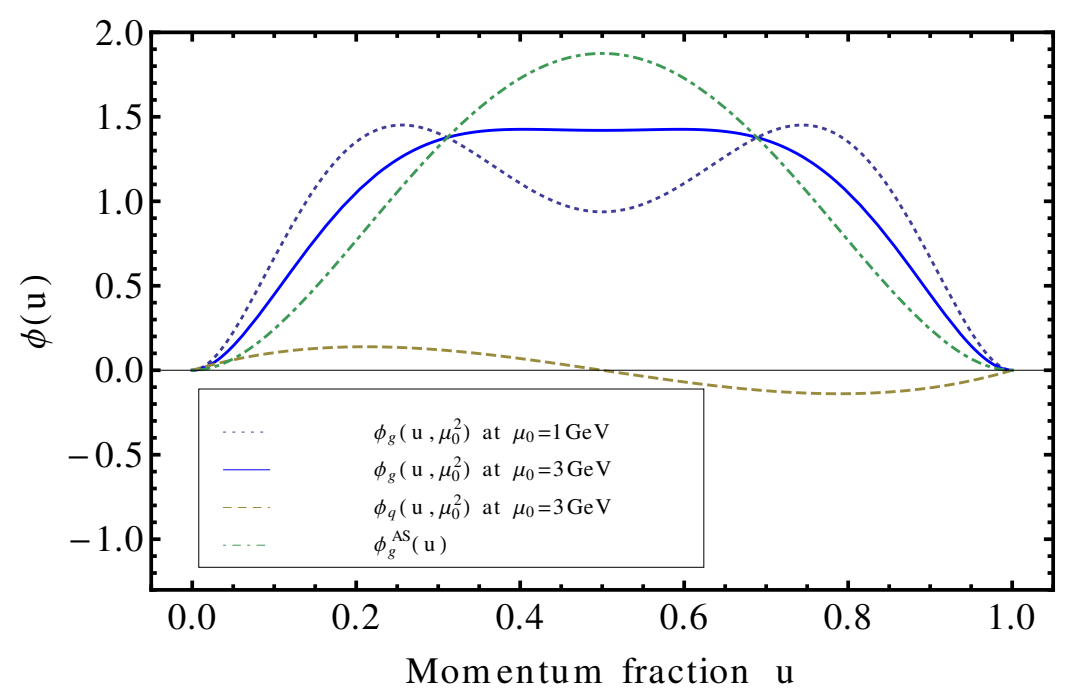

Figure 3. The light-cone distribution amplitude for scalar Glueball, where we only consider the first Gegenbauer momentum and resum the corresponding large logarithms in $a_{3}^{g}(\mu)$ and $a_{1}^{q}(\mu)$, using $\mu_{0}=1 \mathrm{GeV}$ and $a_{3}^{g}\left(\mu_{0}\right)=0.2, a_{1}^{q}\left(\mu_{0}\right)=0$ as input. The asymptotic form of $\phi_{g}(u)$ is $30 u^{2}(1-u)^{2}$.

After solving the renormalizaiton group equation, the resummed Gegenbauer momenta read

$$
\begin{aligned}
& a_{n}^{q}\left(\mu^{2}\right)=\frac{1}{\delta \gamma_{n}^{g q}}\left(a_{n}^{+}\left(\mu_{0}^{2}\right)\left(\lambda_{n}^{+}-\gamma_{n}^{g g}\right)\left[\frac{\alpha_{s}\left(\mu^{2}\right)}{\alpha_{s}\left(\mu_{0}^{2}\right)}\right]^{2 \lambda_{n}^{+} / \beta_{0}}-a_{n}^{-}\left(\mu_{0}^{2}\right)\left(\lambda_{n}^{-}-\gamma_{n}^{g g}\right)\left[\frac{\alpha_{s}\left(\mu^{2}\right)}{\alpha_{s}\left(\mu_{0}^{2}\right)}\right]^{2 \lambda_{n}^{-} / \beta_{0}}\right), \\
& a_{n}^{g}\left(\mu^{2}\right)=\frac{1}{\delta}\left(a_{n}^{+}\left(\mu_{0}^{2}\right)\left[\frac{\alpha_{s}\left(\mu^{2}\right)}{\alpha_{s}\left(\mu_{0}^{2}\right)}\right]^{2 \lambda_{n}^{+} / \beta_{0}}-a_{n}^{-}\left(\mu_{0}^{2}\right)\left[\frac{\alpha_{s}\left(\mu^{2}\right)}{\alpha_{s}\left(\mu_{0}^{2}\right)}\right]^{2 \lambda_{n}^{-} / \beta_{0}}\right),
\end{aligned}
$$

where $\lambda_{n}^{ \pm}$are the eigenvalues

$$
\lambda_{n}^{ \pm}=\frac{1}{2}\left(\gamma_{n}^{g g}+\gamma_{n}^{q q} \pm \delta\right),
$$

with $\delta=\sqrt{\left(\gamma_{n}^{g g}-\gamma_{n}^{q q}\right)^{2}+4 \gamma_{n}^{q g} \gamma_{n}^{g q}}$. And $a_{n}^{ \pm}$are the eigenvectors, with

$$
a_{n}^{ \pm}\left(\mu^{2}\right)=a_{n}^{q}\left(\mu^{2}\right) \gamma_{n}^{g q}-a_{n}^{g}\left(\mu^{2}\right)\left(\lambda_{n}^{\mp}-\gamma_{n}^{g g}\right) .
$$

We show the LCDAs of scalar Glueball in figure 3, where we take $a_{3}^{g}\left(\mu_{0}\right)=0.2$ and $a_{1}^{q}\left(\mu_{0}\right)=0$ with $\mu_{0}=1 \mathrm{GeV}$ as input, and evolute it into another scale.

\section{Phenomenological discussions}

In this section, we will employ the above factorization formulae and analyze the phenomenological results confronting the recent BESIII and CLEO data $[25,26]$. The branching ratio of $J / \psi \rightarrow G+\gamma$ can be written as

$$
\mathcal{B}(J / \psi \rightarrow G+\gamma)=\frac{m_{J / \psi}^{2}-m_{G}^{2}}{16 \pi \Gamma_{J / \psi} m_{J / \psi}^{3}}|\mathcal{M}(J / \psi \rightarrow G+\gamma)|^{2} .
$$


At first, we note that the decay width of $J / \psi \rightarrow G+\gamma$ has been studied by the CLQCD Collaboration within the framework of quenched Lattice QCD [50], which gives

$$
\mathcal{B}(J / \psi \rightarrow G+\gamma)=(3.8 \pm 0.9) \times 10^{-3} .
$$

In the following we will adopt the value of parameters from PDG2014: [51] $m_{J / \psi}=$ $3.0969 \mathrm{GeV}, \Gamma_{J / \psi}=92.9 \mathrm{keV}, m_{\psi(2 S)}=3.686 \mathrm{GeV}, \Gamma_{\psi(2 S)}=286 \mathrm{keV}, m_{\Upsilon}=9.4603 \mathrm{GeV}$, $\Gamma_{\Upsilon}=54.02 \mathrm{keV}, m_{\Upsilon(2 S)}=10.023 \mathrm{GeV}, \Gamma_{\Upsilon(2 S)}=31.98 \mathrm{keV}, m_{\Upsilon(3 S)}=10.355 \mathrm{GeV}$, $\Gamma_{\Upsilon(3 S)}=20.32 \mathrm{keV}$. The values of LDMEs for heavy quarkonia are extracted from their electric widths at NLO as refs. [52, 53], which reads as $\left\langle 0\left|\chi^{\dagger} \boldsymbol{\sigma} \psi\right| J / \psi\right\rangle=0.6408(\mathrm{GeV})^{3 / 2}$, $\left\langle 0\left|\chi^{\dagger} \boldsymbol{\sigma} \psi\right| \psi(2 S)\right\rangle=0.4975(\mathrm{GeV})^{3 / 2},\left\langle 0\left|\chi^{\dagger} \boldsymbol{\sigma} \psi\right| \Upsilon\right\rangle=1.710(\mathrm{GeV})^{3 / 2},\left\langle 0\left|\chi^{\dagger} \boldsymbol{\sigma} \psi\right| \Upsilon(2 S)\right\rangle=$ $1.2502(\mathrm{GeV})^{3 / 2}$ and $\left\langle 0\left|\chi^{\dagger} \boldsymbol{\sigma} \psi\right| \Upsilon(3 S)\right\rangle=1.099(\mathrm{GeV})^{3 / 2}$. The heavy quark mass is adopted as $m_{c}=1.5 \mathrm{GeV}$ and $m_{b}=4.8 \mathrm{GeV}$ [54-56]. From figure 3, one can see that the $\Phi_{q}(u)$ is small, so we will ignore its contribution in the following. We take the mass of scalar Glueball as $m_{G}=1.710 \mathrm{GeV}$ from Lattice QCD [3], then we can extract the decay constant of scalar Glueball, which reads as

$$
f_{g}=0.0386_{-0.0049}^{+0.0097} \mathrm{GeV},
$$

where the uncertainty is from both the Lattice QCD and the running coupling constant. Note that the result is a little smaller than the prediction $(0.10-0.13) \mathrm{GeV}$ from QCD sum rule [38]. We will get a more precise and reliable result for the decay constant of Glueball, if we have the Lattice result for $\Upsilon(n S) \rightarrow G+\gamma$, where the NRQCD $\otimes$ LCDA factorization becomes more solid. We can also predict the branching ratios of scalar Glueball from other vector heavy quarkonia. They are

$$
\begin{aligned}
\mathcal{B}(\psi(2 S) \rightarrow G+\gamma) & =\left(5.9_{-1.4}^{+3.4}\right) \times 10^{-4}, \\
\mathcal{B}(\Upsilon \rightarrow G+\gamma) & =\left(1.3_{-0.3}^{+0.7}\right) \times 10^{-4}, \\
\mathcal{B}(\Upsilon(2 S) \rightarrow G+\gamma) & =\left(1.0_{-0.2}^{+0.6}\right) \times 10^{-4} \\
\mathcal{B}(\Upsilon(3 S) \rightarrow G+\gamma) & =\left(1.2_{-0.3}^{+0.7}\right) \times 10^{-4}
\end{aligned}
$$

Next we will consider the mixing among scalar Glueball and scalar $q \bar{q}$ states. There are many scalar mesons with masses lower than $2 \mathrm{GeV}$, which can be classified into two nonets: one nonet with mass below $1 \mathrm{GeV}$ includes $f_{0}(500), f_{0}(800), K_{0}^{*}(800)$ and $a_{0}(980)$; the other nonet with mass above $1 \mathrm{GeV}$ includes $K_{0}^{*}(1430), a_{0}(1450)$ and two scalar mesons $[18,57]$. One can see that not all three isosinglet scalars $f_{0}(1370), f_{0}(1500)$ and $f_{0}(1710)$ can be accommodated in the $q \bar{q}$ nonet picture at the same time. One of them can have a large possibility of Glueball component. Denoting $n \bar{n}=(u \bar{u}+d \bar{d}) / \sqrt{2}$, we write the mixing formula $[48,49]$

$$
\left|f_{0}^{i}\right\rangle=\alpha_{i}|n \bar{n}\rangle+\beta_{i}|s \bar{s}\rangle+\rho_{i}|G\rangle,
$$

where $f_{0}^{i}$ can be one of $f_{0}(1370), f_{0}(1500)$ and $f_{0}(1710)$.

According to the lattice calculations [58], Lee and Weingarten found that $f_{0}(1710)$ is composed mainly of scalar Glueball. It is reasonable when we take the current experimental data into account. From PDG2014, we find that $\mathcal{B}\left(\psi(J / \psi) \rightarrow f_{0}(1710) \gamma\right)$ is a large value, which will be $1.56 \times 10^{-3}$ when considering $\mathcal{B}\left(\psi(J / \psi) \rightarrow f_{0}(1710) \gamma \rightarrow\right.$ $\pi \bar{\pi} \gamma)=(4.0 \pm 1.0) \times 10^{-4}, \mathcal{B}\left(\psi(J / \psi) \rightarrow f_{0}(1710) \gamma \rightarrow K \bar{K} \gamma\right)=\left(8.5_{-0.9}^{+1.2}\right) \times 10^{-4}$ and $\mathcal{B}\left(\psi(J / \psi) \rightarrow f_{0}(1710) \gamma \rightarrow \omega \bar{\omega} \gamma\right)=(3.1 \pm 1.0) \times 10^{-4}$. On the other hand, the fraction 


\begin{tabular}{|cccccc|}
\hline Branching ratio $\left(10^{-4}\right)$ & This work & LQCD [50] & He et al. [38] & Cheng et al. [57] & PDG2014 [51] \\
\hline $\mathcal{B}\left(J / \psi \rightarrow f_{0}(1370)+\gamma\right)^{a}$ & $9.3 \pm 2.2$ & - & - & - & - \\
$\mathcal{B}\left(J / \psi \rightarrow f_{0}(1500)+\gamma\right)$ & $0.62 \pm 0.15$ & - & - & 2.9 & $1.01 \pm 0.32$ \\
$\mathcal{B}\left(J / \psi \rightarrow f_{0}(1710)+\gamma\right)$ & $28.0 \pm 6.6$ & - & - & 14.5 & $>15.6$ \\
$\mathcal{B}\left(J / \psi \rightarrow G\left(0^{++}\right)+\gamma\right)$ & $38 \pm 9^{b}$ & $38 \pm 9$ & - & - & - \\
$\mathcal{B}\left(\psi(2 S) \rightarrow f_{0}(1370)+\gamma\right)$ & $1.45_{-0.34}^{+0.83}$ & - & - & - & - \\
$\mathcal{B}\left(\psi(2 S) \rightarrow f_{0}(1500)+\gamma\right)$ & $0.97_{-0.23}^{+0.56}$ & - & - & - & - \\
$\mathcal{B}\left(\psi(2 S) \rightarrow f_{0}(1710)+\gamma\right)$ & $4.4_{-1.0}^{+2.5}$ & - & - & - & $>0.9$ \\
$\mathcal{B}\left(\psi(2 S) \rightarrow G\left(0^{++}\right)+\gamma\right)$ & $5.9_{-1.4}^{+3.4}$ & - & - & - & - \\
$\mathcal{B}\left(\Upsilon \rightarrow f_{0}(1370)+\gamma\right)$ & $0.32_{-0.08}^{+0.18}$ & - & 4.8 & - & - \\
$\mathcal{B}\left(\Upsilon \rightarrow f_{0}(1500)+\gamma\right)$ & $0.021_{-0.005}^{+0.012}$ & - & 4.2 & - & $<0.15$ \\
$\mathcal{B}\left(\Upsilon \rightarrow f_{0}(1710)+\gamma\right)$ & $0.96_{-0.23}^{+0.55}$ & - & 1.5 & - & $<2.6$ \\
$\mathcal{B}\left(\Upsilon \rightarrow G\left(0^{++}\right)+\gamma\right)$ & $1.3_{-0.3}^{+0.7}$ & - & - & - & $<2.6$ \\
$\mathcal{B}\left(\Upsilon(2 S) \rightarrow f_{0}(1370)+\gamma\right)$ & $0.26_{-0.06}^{+0.14}$ & - & - & - & - \\
$\mathcal{B}\left(\Upsilon(2 S) \rightarrow f_{0}(1500)+\gamma\right)$ & $0.016_{-0.004}^{+0.009}$ & - & - & - & - \\
$\mathcal{B}\left(\Upsilon(2 S) \rightarrow f_{0}(1710)+\gamma\right)$ & $0.77_{-0.18}^{+0.44}$ & - & - & - & $<5.9$ \\
$\mathcal{B}\left(\Upsilon(2 S) \rightarrow G\left(0^{++}\right)+\gamma\right)$ & $1.0_{-0.2}^{+0.6}$ & - & - & - & $<5.9$ \\
$\mathcal{B}\left(\Upsilon(3 S) \rightarrow f_{0}(1370)+\gamma\right)$ & $0.30_{-0.07}^{+0.17}$ & - & - & - & - \\
$\mathcal{B}\left(\Upsilon(3 S) \rightarrow f_{0}(1500)+\gamma\right)$ & $0.019_{-0.005}^{+0.010}$ & - & - & - & - \\
$\mathcal{B}\left(\Upsilon(3 S) \rightarrow f_{0}(1710)+\gamma\right)$ & $0.90_{-0.21}^{+0.51}$ & - & - & - & - \\
$\mathcal{B}\left(\Upsilon(3 S) \rightarrow G\left(0^{++}\right)+\gamma\right)$ & $1.2_{-0.3}^{+0.7}$ & - & - & - & - \\
\hline
\end{tabular}

${ }^{a}$ Here we adopt the mixing matrix as eq. (4.6) from Lattice QCD, however the mixing matrix elements for $f_{0}(1370)$ we think still need to be tested further.

${ }^{b}$ We use the Lattice QCD result to extract the decay constant of Glueball.

Table 1. The branching ratios $\left(10^{-4}\right)$ of $V\left(1^{--}\right) \rightarrow f_{0}^{i}+\gamma$ and $V\left(1^{--}\right) \rightarrow G\left(0^{++}\right)+\gamma$, where $\mathrm{V}$ denotes one of heavy quarkonium $J / \psi, \psi(2 S)$ and $\Upsilon(n S), f_{0}^{i}$ denotes one of $f_{0}(1370), f_{0}(1500)$ and $f_{0}(1710)$.

$\mathcal{B}\left(\psi(J / \psi) \rightarrow f_{0}(1710) \gamma\right) / \mathcal{B}\left(\psi(J / \psi) \rightarrow f_{0}(1500) \gamma\right)$ is around order of 10 , and $f_{0}(1370)$ is still not observed in the $J \psi$ radiative decays. The mixing matrix can be expressed as [58]

$$
\left(\begin{array}{c}
f_{0}(1370) \\
f_{0}(1500) \\
f_{0}(1710)
\end{array}\right)=\left(\begin{array}{ccc}
0.819(89) & 0.290(91) & -0.495(118) \\
-0.399(113) & 0.908(37) & -0.128(52) \\
0.413(87) & 0.302(52) & 0.859(54)
\end{array}\right)\left(\begin{array}{c}
|n \bar{n}\rangle \\
|s \bar{s}\rangle \\
|G\rangle
\end{array}\right) .
$$

One can easily see that $f_{0}(1370)$ has a large possibility of the $n \bar{n}$ component, while $f_{0}(1500)$ is dominated by the $s \bar{s}$ component.

We assume that Glueball component dominates the contribution in $V \rightarrow f_{0}^{i}+\gamma$ since the processes from $q \bar{q}$ components are suppressed by the strong coupling squared $\alpha_{s}^{2}$. Here we simply generalize it to all the three scalar mesons. The related branching ratios are given in table 1, where one can see that our results are comparable with data from PDG2014 except the predictions for $f_{0}(1370)$. Employing the mixing matrix elements of eq. (4.6) 
based on Lattice QCD, we predict a large branching ratio for $V\left(1^{--}\right) \rightarrow f_{0}(1370)+\gamma$ while there is no signal at experiment. We conclude that the first line in the matrix of eq. (4.6) may be not precise enough and it need to be checked by the following experiment.

\section{Conclusion}

In this paper, we have established the factorization formulae for heavy vector quarkonium radiative decays into scalar Glueball, by studying one-loop corrections to the hard kernel, LDMEs of heavy quarkonium, and LCDA of scalar Glueball. The NRQCDQLCDA factorization formulae shall be valid to all orders of the strong coupling constant $\alpha_{s}$ in the leading-order twist and heavy quark velocity, after considering a two-dimensional LCDA of Glueball which is defined by non-local light-cone gauge-invariant operators matrix elements. The universality of LCDA for Glueball ensures us to extract its decay constant. Matching to the CLQCD results, we have extracted the decay constant for scalar Glueball, i.e. $f_{g}=0.0386_{-0.0049}^{+0.0097} \mathrm{GeV}$. We also predict the branching ratios of scalar Glueball from other heavy vector quarkonia such as $\psi(2 S)$ and $\Upsilon(n S)$, which can be checked in the upcoming experiment. The factorization formulae can also be applied to pseudoscalar and tensor Glueball production from heavy vector quarkonia. A systematic study on Glueball production and decay with different quantum numbers shall be investigated in order to hunting and identifying Glueball with a large confidence. We will address these issues in following studies.

\section{Acknowledgments}

I greatly thank Profs. Xiangdong Ji, Cong-Feng Qiao, Feng Yuan, Jian-Ping Ma, and Chengping Shen for fruitful discussions. I also specially thank Wei Wang for pointing out the phenomenological analyses. This work was supported in part by a key laboratory grant from the Office of Science and Technology, Shanghai Municipal Government (No. 11DZ2260700), by Shanghai Natural Science Foundation under Grant No. 15ZR1423100, and by the Open Project Program of State Key Laboratory of Theoretical Physics, Institute of Theoretical Physics, Chinese Academy of Sciences, China (No. Y5KF111CJ1).

\section{A Short-distance coefficients for hard kernels}

In the appendix, we give the explicit results of the short-distance coefficients for hard kernels.

$$
\begin{aligned}
H_{0}^{a}= & {\left[\frac{(2 u-1) \log (u)}{2(u-1)} \log \frac{\mu^{2}}{m_{c}^{2}}-\frac{(2 u-1) N_{c}\left(\operatorname{Li}_{2}(1-2 u)+\log (u)(\log (u)-2+2 \log (2))\right)}{6(u-1)}\right.} \\
& +\frac{(2 u-1)\left(54-\left(\pi^{2}-18-2 f_{1}^{a}\right) N_{c}\right)}{144(u-1) u}+\frac{1}{8(u-1) u}\left(( 2 u - 1 ) \left(3 B_{1}-2 B_{2}+3 C_{1}\right.\right. \\
& \left.\left.\left.-\left(8 u^{2}-8 u+3\right) C_{2}\right)+2\left(8 u^{2}-9 u+3\right) B_{3}\right)\right]-u \rightarrow(1-u), \\
H_{1}^{a}= & H_{0}^{a},
\end{aligned}
$$




$$
\begin{aligned}
H_{0}^{b}= & {\left[\frac{\left(2 u^{2}-2 u+1\right) N_{c} \log (u)}{u-1} \log \frac{\mu^{2}}{m_{c}^{2}}-\frac{\left(2 u^{2}-2 u+1\right) N_{c}}{u-1}\left(\operatorname{Li}_{2}(1-2 u)+\log (u)(\log (u)-2\right.\right.} \\
& \left.+2 \log (2))-\frac{f_{1}^{a}}{12 u}\right)+\frac{2 f_{1}^{b}+b_{1}^{b} B_{1}}{96(u-1)^{2} u^{2}}+\frac{b_{2}^{b} B_{2}}{96(u-1) u}+\frac{b_{3}^{b} B_{3}}{48(u-1) u^{2}(2 u-1)}+\frac{b_{4}^{b} B_{4}}{12(u-1)^{2}} \\
& +\frac{c_{1}^{b} C_{1}}{192(1-2 u)^{4}(u-1)^{2} u^{2}}+\frac{c_{2}^{b} C_{2}}{48(u-1)^{2} u^{2}}+\frac{c_{3}^{b} C_{3}}{32(1-2 u)^{4}(u-1) u^{2}}+\frac{c_{4}^{b} C_{4}}{24(u-1)^{2} u} \\
& +\frac{c_{5}^{b} C_{5}}{96(1-2 u)^{4} u^{2}}+\frac{c_{6}^{b} C_{6}}{48(1-2 u)^{4}(u-1)^{2}}-\frac{\left(u^{4}-2 u^{3}+u^{2}-3\right) C_{F}}{2(u-1)^{2} u^{2}} C_{7} \\
& \left.-\frac{\left(2 u^{3}-u^{2}-9 u+1\right) C_{F}}{4(u-1) u^{2}} C_{8}\right]+u \rightarrow(1-u),
\end{aligned}
$$

where

$$
\begin{aligned}
f_{1}^{a}= & 12\left(\operatorname{Li}_{2}(1-2 u)+\operatorname{Li}_{2}(2 u)+\log (1-2 u)(\log (u)+\log (2))\right)-\pi^{2}, \\
f_{1}^{b}= & \left(\left(438-4 \pi^{2}\right) u^{4}+\left(8 \pi^{2}-876\right) u^{3}+\left(585-6 \pi^{2}\right) u^{2}+\left(2 \pi^{2}-147\right) u-6\right) N_{c} \\
& -12\left(52 u^{4}-104 u^{3}+46 u^{2}+6 u-3\right) C_{F}-90 u^{4}+180 u^{3}-433 u^{2}+343 u-36, \\
b_{1}^{b}= & 3\left(180 u^{4}-360 u^{3}+237 u^{2}-57 u-2\right) N_{c}+12\left(2 u^{4}-4 u^{3}+8 u^{2}-6 u+3\right) C_{F} \\
& -1172 u^{4}+2344 u^{3}-1587 u^{2}+415 u-36, \\
b_{2}^{b}= & -15(1-2 u)^{2} N_{c}+12 C_{F}-124 u^{2}+124 u-45, \\
b_{3}^{b}= & 3(1-2 u)^{2}\left(48 u^{3}-92 u^{2}+63 u-10\right) N_{c}+12\left(6 u^{4}-37 u^{3}+47 u^{2}-21 u+3\right) C_{F} \\
& +1312 u^{4}-2588 u^{3}+1740 u^{2}-455 u+36, \\
b_{4}^{b}= & \left(6 u^{2}-6 u+9\right) N_{c}-3\left(4 u^{2}+3 u-1\right) C_{F}-2 u^{2}+3 u-1, \\
c_{1}^{b}= & 3\left(1792 u^{8}-7168 u^{7}+12688 u^{6}-12976 u^{5}+8368 u^{4}-3472 u^{3}+895 u^{2}-127 u+8\right) N_{c} \\
& +4(u-1) u(1-2 u)^{4}\left(51 C_{F}-40 u^{2}+40 u-1\right), \\
c_{2}^{b}= & -3\left(16 u^{4}-32 u^{3}+27 u^{2}-11 u+2\right)(1-2 u)^{2} N_{c}+6(1-2 u)^{2} C_{F} \\
& +u\left(100 u^{3}-200 u^{2}+117 u-17\right), \\
c_{3}^{b}= & u\left(-128 u^{7}+184 u^{6}+932 u^{5}-2372 u^{4}+2224 u^{3}-1039 u^{2}+246 u-23\right) N_{c} \\
& -4(1-2 u)^{4}\left(3 u^{3}-4 u^{2}-5 u-1\right) C_{F}, \\
c_{4}^{b}= & 3\left(10 u^{3}-24 u^{2}+8 u+7\right) C_{F}+u\left(-3\left(u^{2}-3 u+4\right) N_{c}-32 u^{3}+37 u^{2}+4 u-9\right), \\
c_{5}^{b}= & 3\left(128 u^{7}-648 u^{6}+1356 u^{5}-1256 u^{4}+472 u^{3}-3 u^{2}-40 u+8\right) N_{c} \\
& +12\left(3 u^{2}-20 u+24\right)(1-2 u)^{4} C_{F}-4 u(9 u+2)(1-2 u)^{4}, \\
c_{6}^{b}= & (u-1)\left(3\left(128 u^{6}-56 u^{5}-220 u^{4}+232 u^{3}-72 u^{2}+7 u+1\right) N_{c}+16(u-1)(1-2 u)^{4}\right) \\
& -12(1-2 u)^{4}\left(3 u^{2}+7 u+16\right) C_{F}, \\
& (\mathrm{~A} .4) \\
& (1)
\end{aligned}
$$

and

$$
\begin{aligned}
H_{1}^{b}= & H_{0}^{b}+\left[\frac{\tilde{c}_{1}^{b} C_{1}}{96(1-2 u)^{4}(u-1) u}+\frac{\tilde{c}_{3}^{b} C_{3}}{96(1-2 u)^{4}(u-1) u}+\frac{\tilde{c}_{4}^{b} C_{4}}{24(u-1) u}+\frac{\tilde{c}_{5}^{b} C_{5}}{48(1-2 u)^{4} u^{2}}\right. \\
& +\frac{\tilde{c}_{6}^{b} C_{6}}{24(1-2 u)^{4}(u-1)^{2}}-\frac{\left(2 u^{4}-4 u^{3}+u^{2}+u-3\right) C_{F}}{(u-1)^{2} u^{2}} C_{7} \\
& \left.+\frac{\left(4 u^{2}-3 u+5\right) C_{F}}{2(u-1) u} C_{8}+u \rightarrow(1-u)\right],
\end{aligned}
$$




$$
\begin{aligned}
\tilde{c}_{1}^{b}= & -3\left(352 u^{6}-1056 u^{5}+1752 u^{4}-1744 u^{3}+992 u^{2}-296 u+41\right) N_{c} \\
& +24\left(4 u^{2}-4 u+3\right)(1-2 u)^{4} C_{F}+4\left(16 u^{2}-16 u+45\right)(1-2 u)^{4}, \\
\tilde{c}_{3}^{b}= & 12(1-2 u)^{4}\left(2 u^{2}+3 u+1\right) C_{F}-(u-1)\left(3 \left(176 u^{6}-496 u^{5}+796 u^{4}\right.\right. \\
& \left.\left.-792 u^{3}+456 u^{2}-129 u+15\right) N_{c}-4(1-2 u)^{4}\left(8 u^{2}-5 u-3\right)\right), \\
\tilde{c}_{4}^{b}= & u\left(-3(4 u-5) N_{c}+32 u^{2}-28 u-45\right)-12\left(2 u^{3}-5 u^{2}+3\right) C_{F}, \\
\tilde{c}_{5}^{b}= & u\left(3\left(176 u^{6}-560 u^{5}+988 u^{4}-1016 u^{3}+584 u^{2}-183 u+28\right) N_{c}\right. \\
& \left.-2(1-2 u)^{4}\left(16 u^{2}+10 u-55\right)\right)+12\left(4 u^{2}-15 u+12\right)(1-2 u)^{4} C_{F}, \\
\tilde{c}_{6}^{b}= & (u-1)\left(3\left(176 u^{6}-496 u^{5}+796 u^{4}-792 u^{3}+456 u^{2}-129 u+15\right) N_{c}\right. \\
& \left.-4(1-2 u)^{4}\left(8 u^{2}-5 u-3\right)\right)-72(1-2 u)^{4}\left(u^{2}+1\right) C_{F} .
\end{aligned}
$$

The coefficients $b_{i}, c_{i}$ and $\tilde{c}_{i}$ are related to the scalar Passarino-Veltman integrals defined in refs. $[59,60]$, and here we have the relation $C_{i}=m_{c}^{2} C_{i}^{0}$ :

$$
\begin{aligned}
& B_{1}=B_{0}\left(0, m_{c}^{2}, m_{c}^{2}\right), \\
& B_{2}=B_{0}\left(-m_{c}^{2}, 0, m_{c}^{2}\right), \\
& B_{3}=B_{0}\left((1-2 u) m_{c}^{2}, 0, m_{c}^{2}\right), \\
& B_{4}=B_{0}\left(4 u m_{c}^{2}, m_{c}^{2}, m_{c}^{2}\right) \\
& C_{1}^{0}=\mathrm{C}_{0}\left(-m_{c}^{2}, m_{c}^{2}, 0, m_{c}^{2}, 0, m_{c}^{2}\right), \\
& C_{2}^{0}=\mathrm{C}_{0}\left(0,(1-2 u) m_{c}^{2},(2 u-1) m_{c}^{2}, m_{c}^{2}, m_{c}^{2}, 0\right), \\
& C_{3}^{0}=\mathrm{C}_{0}\left(m_{c}^{2}, 4 u m_{c}^{2},(2 u-1) m_{c}^{2}, 0, m_{c}^{2}, m_{c}^{2}\right), \\
& C_{4}^{0}=\mathrm{C}_{0}\left(m_{c}^{2}, 0,(1-2 u) m_{c}^{2}, 0, m_{c}^{2}, m_{c}^{2}\right) \\
& C_{5}^{0}=\mathrm{C}_{0}\left(-m_{c}^{2}, 0,(1-2 u) m_{c}^{2}, 0, m_{c}^{2}, m_{c}^{2}\right), \\
& C_{6}^{0}=\mathrm{C}_{0}\left(0,0,4 u m_{c}^{2}, m_{c}^{2}, m_{c}^{2}, m_{c}^{2}\right), \\
& C_{7}^{0}=\mathrm{C}_{0}\left(4 m_{c}^{2}, 0,0, m_{c}^{2}, m_{c}^{2}, m_{c}^{2}\right), \\
& C_{8}^{0}=\mathrm{C}_{0}\left(4 m_{c}^{2}, 0,4 u m_{c}^{2}, m_{c}^{2}, m_{c}^{2}, m_{c}^{2}\right) .
\end{aligned}
$$

Open Access. This article is distributed under the terms of the Creative Commons Attribution License (CC-BY 4.0), which permits any use, distribution and reproduction in any medium, provided the original author(s) and source are credited.

\section{References}

[1] APE collaboration, M. Albanese et al., Glueball Masses and String Tension in Lattice QCD, Phys. Lett. B 192 (1987) 163 [inSPIRE].

[2] C.J. Morningstar and M.J. Peardon, The Glueball spectrum from an anisotropic lattice study, Phys. Rev. D 60 (1999) 034509 [hep-lat/9901004] [INSPIRE].

[3] Y. Chen et al., Glueball spectrum and matrix elements on anisotropic lattices, Phys. Rev. D 73 (2006) 014516 [hep-lat/0510074] [INSPIRE].

[4] E. Gregory et al., Towards the glueball spectrum from unquenched lattice QCD, JHEP 10 (2012) 170 [arXiv:1208.1858] [INSPIRE]. 
[5] E. Bagan and T.G. Steele, Mass of the Scalar Glueball: Higher Loop Effects in the QCD Sum Rules, Phys. Lett. B 243 (1990) 413 [InSPIRE].

[6] J.-P. Liu and D.-H. Liu, The leading quark mass corrections to the QCD sum rules for the O++ scalar glueball, J. Phys. G 19 (1993) 373 [inSPIRE].

[7] W. Shuiguo, Z. Zhenyu and L. Jueping, $0^{++}$scalar glueball in finite-width Gaussian sum rules, Phys. Rev. D 82 (2010) 016003 [arXiv: 1007.2465] [INSPIRE].

[8] T. Huang, H.-Y. Jin and A.-L. Zhang, Determination of the scalar glueball mass in QCD sum rules, Phys. Rev. D 59 (1999) 034026 [hep-ph/9807391] [INSPIRE].

[9] C.-F. Qiao and L. Tang, Finding the $0^{--}$Glueball, Phys. Rev. Lett. 113 (2014) 221601 [arXiv: 1408.3995] [INSPIRE].

[10] X.-H. Yuan and L. Tang, Fermion correction to the mass of the scalar glueball in QCD sum rule, Commun. Theor. Phys. 54 (2010) 495 [arXiv:0911.0806] [InSPIRE].

[11] G. Hao, C.-F. Qiao and A.-L. Zhang, $0-+$ trigluon glueball and its implication for a recent BES observation, Phys. Lett. B 642 (2006) 53 [hep-ph/0512214] [INSPIRE].

[12] C. Csáki, H. Ooguri, Y. Oz and J. Terning, Glueball mass spectrum from supergravity, JHEP 01 (1999) 017 [hep-th/9806021] [INSPIRE].

[13] F. Brünner, D. Parganlija and A. Rebhan, Glueball Decay Rates in the Witten-Sakai-Sugimoto Model, Phys. Rev. D 91 (2015) 106002 [arXiv:1501.07906] [INSPIRE].

[14] F. Brünner and A. Rebhan, Nonchiral enhancement of scalar glueball decay in the Witten-Sakai-Sugimoto model, arXiv: 1504.05815 [INSPIRE].

[15] D. Parganlija, Scalar Glueball in a Top-Down Holographic Approach to QCD, Acta Phys. Polon. Supp. 8 (2015) 219 [arXiv: 1503.00550] [INSPIRE].

[16] J. Sonnenschein and D. Weissman, Glueballs as rotating folded closed strings, arXiv: 1507.01604 [INSPIRE].

[17] C.E. Carlson, T.H. Hansson and C. Peterson, Meson, Baryon and Glueball Masses in the MIT Bag Model, Phys. Rev. D 27 (1983) 1556 [INSPIRE].

[18] H.-Y. Cheng, C.-K. Chua and K.-F. Liu, Revisiting Scalar Glueballs, arXiv:1503.06827 [INSPIRE].

[19] X.-G. He and T.-C. Yuan, Glueball Production via Gluonic Penguin B Decays, Eur. Phys. J. C 75 (2015) 136 [arXiv:1503.03577] [INSPIRE].

[20] W. Wang, Y.-L. Shen and C.-D. Lu, B-to-Glueball form factor and Glueball production in B decays, J. Phys. G 37 (2010) 085006 [arXiv:0908.2216] [INSPIRE].

[21] M. Chanowitz, Chiral suppression of scalar glueball decay, Phys. Rev. Lett. 95 (2005) 172001 [hep-ph/0506125] [INSPIRE].

[22] K.-T. Chao, X.-G. He and J.-P. Ma, Comment on 'Chiral suppression of scalar glueball decay', Phys. Rev. Lett. 98 (2007) 149103 [arXiv:0704.1061] [INSPIRE].

[23] BES collaboration, X.-y. Shen, J/psi radiative decays, eConf C 020620 (2002) THAT07 [hep-ex/0209031] [INSPIRE].

[24] BES collaboration, J.Z. Bai et al., Partial wave analyses of $J / \vec{\psi} \gamma K^{+} K^{-}$and $\gamma K_{S}^{0} K_{S}^{0}$, Phys. Rev. D 68 (2003) 052003 [hep-ex/0307058] [INSPIRE]. 
[25] BESIII collaboration, M. Ablikim et al., Partial wave analysis of $J / \psi \rightarrow \gamma \eta \eta$, Phys. Rev. D 87 (2013) 092009 [arXiv: 1301.0053] [INSPIRE].

[26] S. Dobbs, A. Tomaradze, T. Xiao and K.K. Seth, Comprehensive Study of the Radiative Decays of $J / \psi$ and $\psi(2 S)$ to Pseudoscalar Meson Pairs and Search for Glueballs, Phys. Rev. D 91 (2015) 052006 [arXiv:1502.01686] [INSPIRE].

[27] G.P. Lepage and S.J. Brodsky, Exclusive Processes in Perturbative Quantum Chromodynamics, Phys. Rev. D 22 (1980) 2157 [InSPIRE].

[28] A.V. Efremov and A.V. Radyushkin, Factorization and Asymptotical Behavior of Pion Form-Factor in QCD, Phys. Lett. B 94 (1980) 245 [InSPIRE].

[29] A. Duncan and A.H. Mueller, Asymptotic Behavior of Composite Particle Form-Factors and the Renormalization Group, Phys. Rev. D 21 (1980) 1636 [INSPIRE].

[30] X.-D. Ji, Deeply virtual Compton scattering, Phys. Rev. D 55 (1997) 7114 [hep-ph/9609381] [INSPIRE].

[31] X.-D. Ji, Off forward parton distributions, J. Phys. G 24 (1998) 1181 [hep-ph/9807358] [INSPIRE].

[32] A.V. Belitsky and D. Mueller, Broken conformal invariance and spectrum of anomalous dimensions in QCD, Nucl. Phys. B 537 (1999) 397 [hep-ph/9804379] [INSPIRE].

[33] X.-d. Ji and F. Yuan, Parton distributions in light cone gauge: Where are the final state interactions?, Phys. Lett. B 543 (2002) 66 [hep-ph/0206057] [INSPIRE].

[34] M. Burkardt, X.-d. Ji and F. Yuan, Scale dependence of hadronic wave functions and parton densities, Phys. Lett. B 545 (2002) 345 [hep-ph/0205272] [INSPIRE].

[35] S. Fleming and A.K. Leibovich, Flavor-singlet light-cone amplitudes and radiative Upsilon decays in SCET, Phys. Rev. D 70 (2004) 094016 [hep-ph/0407259] [INSPIRE].

[36] S. Fleming, C. Lee and A.K. Leibovich, Exclusive radiative decays of Upsilon in SCET, Phys. Rev. D 71 (2005) 074002 [hep-ph/0411180] [INSPIRE].

[37] M.B. Cakir and G.R. Farrar, Radiative decay of vector quarkonium: Constraints on glueballs and light gluinos, Phys. Rev. D 50 (1994) 3268 [hep-ph/9402203] [INSPIRE].

[38] X.G. He, H.Y. Jin and J.P. Ma, Radiative decay of upsilon into a scalar glueball, Phys. Rev. D 66 (2002) 074015 [hep-ph/0203191] [INSPIRE].

[39] M. Melis, F. Murgia and J. Parisi, Glueball production in radiative $J / \psi, \Upsilon$ decays, Phys. Rev. D 70 (2004) 034021 [hep-ph/0404070] [inSPIRE].

[40] J.P. Ma, A QCD analysis for radiative decays of upsilon into f(2)(1270), Nucl. Phys. B 605 (2001) 625 [Erratum ibid. B 611 (2001) 523] [hep-ph/0103237] [INSPIRE].

[41] G.T. Bodwin, E. Braaten and G.P. Lepage, Rigorous QCD analysis of inclusive annihilation and production of heavy quarkonium, Phys. Rev. D 51 (1995) 1125 [Erratum ibid. D 55 (1997) 5853] [hep-ph/9407339] [INSPIRE].

[42] M.K. Chase, The $Q^{2}$ evolution of flavor singlet wave functions in $Q C D$, Nucl. Phys. B 174 (1980) 109 [INSPIRE].

[43] A.V. Belitsky and D. Mueller, Next-to-leading order evolution of twist-2 conformal operators: The Abelian case, Nucl. Phys. B 527 (1998) 207 [hep-ph/9802411] [InSPIRE]. 
[44] Y.-Q. Ma, J.-W. Qiu and H. Zhang, Heavy quarkonium fragmentation functions from a heavy quark pair. I. S wave, Phys. Rev. D 89 (2014) 094029 [arXiv:1311.7078] [InSPIRE].

[45] Y.-Q. Ma, J.-W. Qiu and H. Zhang, Heavy quarkonium fragmentation functions from a heavy quark pair. II. P wave, Phys. Rev. D 89 (2014) 094030 [arXiv:1401.0524] [INSPIRE].

[46] X.-P. Wang and D. Yang, The leading twist light-cone distribution amplitudes for the S-wave and $P$-wave quarkonia and their applications in single quarkonium exclusive productions, JHEP 06 (2014) 121 [arXiv: 1401.0122] [INSPIRE].

[47] P. Kroll and K. Passek-Kumericki, The two gluon components of the eta and eta-prime mesons to leading twist accuracy, Phys. Rev. D 67 (2003) 054017 [hep-ph/0210045] [INSPIRE].

[48] F.E. Close and Q. Zhao, Production of $f_{0}(1710), f_{0}(1500)$ and $f_{0}(1370)$ in $J / \psi$ hadronic decays, Phys. Rev. D 71 (2005) 094022 [hep-ph/0504043] [INSPIRE].

[49] Y.M. Cho, X.Y. Pham, P. Zhang, J.-J. Xie and L.-P. Zou, Glueball Physics in QCD, Phys. Rev. D 91 (2015) 114020 [arXiv:1503.08890] [InSPIRE].

[50] CLQCD collaboration, L.-C. Gui et al., Scalar Glueball in Radiative J/ $\psi$ Decay on the Lattice, Phys. Rev. Lett. 110 (2013) 021601 [arXiv:1206.0125] [INSPIRE].

[51] Particle Data Group collaboration, K.A. Olive et al., Review of Particle Physics, Chin. Phys. C 38 (2014) 090001.

[52] R. Zhu, The Exclusive Decay of Upsilon into $h_{c}$, the $X(3940)$ and $X(4160)$, arXiv: 1507.02031 [INSPIRE].

[53] C.-F. Qiao, P. Sun, D. Yang and R.-L. Zhu, $B_{c}$ exclusive decays to charmonium and a light meson at next-to-leading order accuracy, Phys. Rev. D 89 (2014) 034008 [arXiv:1209.5859] [INSPIRE].

[54] C.-F. Qiao and R.-L. Zhu, Understanding the cross section of $e^{+} e^{-} \rightarrow \eta J / \psi$ process via nonrelativistic QCD, Phys. Rev. D 89 (2014) 074006 [arXiv: 1403.1918] [INSPIRE].

[55] C.-F. Qiao and R.-L. Zhu, Estimation of semileptonic decays of $B_{c}$ meson to S-wave charmonia with nonrelativistic QCD, Phys. Rev. D 87 (2013) 014009 [arXiv:1208.5916] [INSPIRE].

[56] W. Wang and R.-L. Zhu, Radiative leptonic $B_{c} \rightarrow \gamma \ell \bar{\nu}$ decay in effective field theory beyond leading order, Eur. Phys. J. C 75 (2015) 360 [arXiv:1501.04493] [INSPIRE].

[57] H.-Y. Cheng, C.-K. Chua and K.-F. Liu, Scalar glueball, scalar quarkonia and their mixing, Phys. Rev. D 74 (2006) 094005 [hep-ph/0607206] [INSPIRE].

[58] W.-J. Lee and D. Weingarten, Scalar quarkonium masses and mixing with the lightest scalar glueball, Phys. Rev. D 61 (2000) 014015 [hep-lat/9910008] [INSPIRE].

[59] G. Passarino and M.J.G. Veltman, One Loop Corrections for $e^{+} e^{-}$Annihilation Into $\mu^{+} \mu^{-}$ in the Weinberg Model, Nucl. Phys. B 160 (1979) 151 [INSPIRE].

[60] T. Hahn and M. Pérez-Victoria, Automatized one loop calculations in four-dimensions and D-dimensions, Comput. Phys. Commun. 118 (1999) 153 [hep-ph/9807565] [INSPIRE]. 